Eur Neurol 2003;49:249-251

DOI: $10.1159 / 000070198$

\section{Nonionic Contrast Neurotoxicity after Coronary Angiography Mimicking Subarachnoid Hemorrhage}

Joachim Veldena, Petra Milz ${ }^{\mathrm{b}}$, Frank Winklera, Klaus Seelos ${ }^{\mathrm{b}}$, Gerhard F. Hamann ${ }^{\text {a }}$

a Department of Neurology and ${ }^{b}$ Institute of Radiology, Division of Neuroradiology, Klinikum Grosshadern, Ludwig Maximilian University, Munich, Germany

\section{Introduction}

The incidence of neurotoxic events due to intravascular application of iodinated $\mathrm{x}$-ray contrast media $(\mathrm{CM})$ has decreased markedly since the introduction of nonionic CM [1]. Severe neurotoxic complications are accompanied by disruption of the blood-brain barrier and $\mathrm{CM}$ extravasation [1-6]. Coronary angiography involving only nonionic $\mathrm{CM}$ has been reported to cause transient cortical blindness, confusion and amnesia, but no focal deficits [3, 4], whereas coronary angiography involving ionic $\mathrm{CM}$ was followed by more severe complications including seizures, motor and speech deficits [5, 6]. Here we report on a patient who developed transient hemiparesis, aphasia and confusion due to neurotoxicity of nonionic $\mathrm{CM}$ after coronary angiography, and whose CT findings mimicked subarachnoid hemorrhage (SAH).

\section{Case Report}

An 82-year-old right-handed Caucasian woman with progressive angina underwent transfemoral coronary angioplasty with stent placement. Her medical history included arterial hypertension and unilateral nephrectomy, but no neurological antecedents. Baseline serum creatinine levels were marginally elevated at $106 \mu M$, reflecting $~ 50 \%$ impairment in glomerular filtration. The angiographic intervention took $134 \mathrm{~min}$ and required the injection of $500 \mathrm{ml}$ of iomeprol (Iomeron ${ }^{\mathrm{TM}} 350$, Bracco SpA, Italy), a monomeric nonionic CM. Additional treatment included 7,500 IU heparin, $300 \mathrm{mg}$ clopidogrel and $100 \mathrm{mg}$ aspirin. Before and during the intervention, the patient had no neurological deficits. However, $30 \mathrm{~min}$ after the
Fig. 1. Time series of brain CT scans from a patient with nonionic contrast neurotoxicity after coronary angiography. Bihemispheric sulcal subarachnoid contrast enhancements at $6 \mathrm{~h}(\mathbf{a})$ and $12 \mathrm{~h}(\mathbf{b})$, fading at $21 \mathrm{~h}(\mathbf{c})$, and completely cleared at $45 \mathrm{~h}$ (d). Note head rotation to the left (a) corresponding to right hemiparesis.
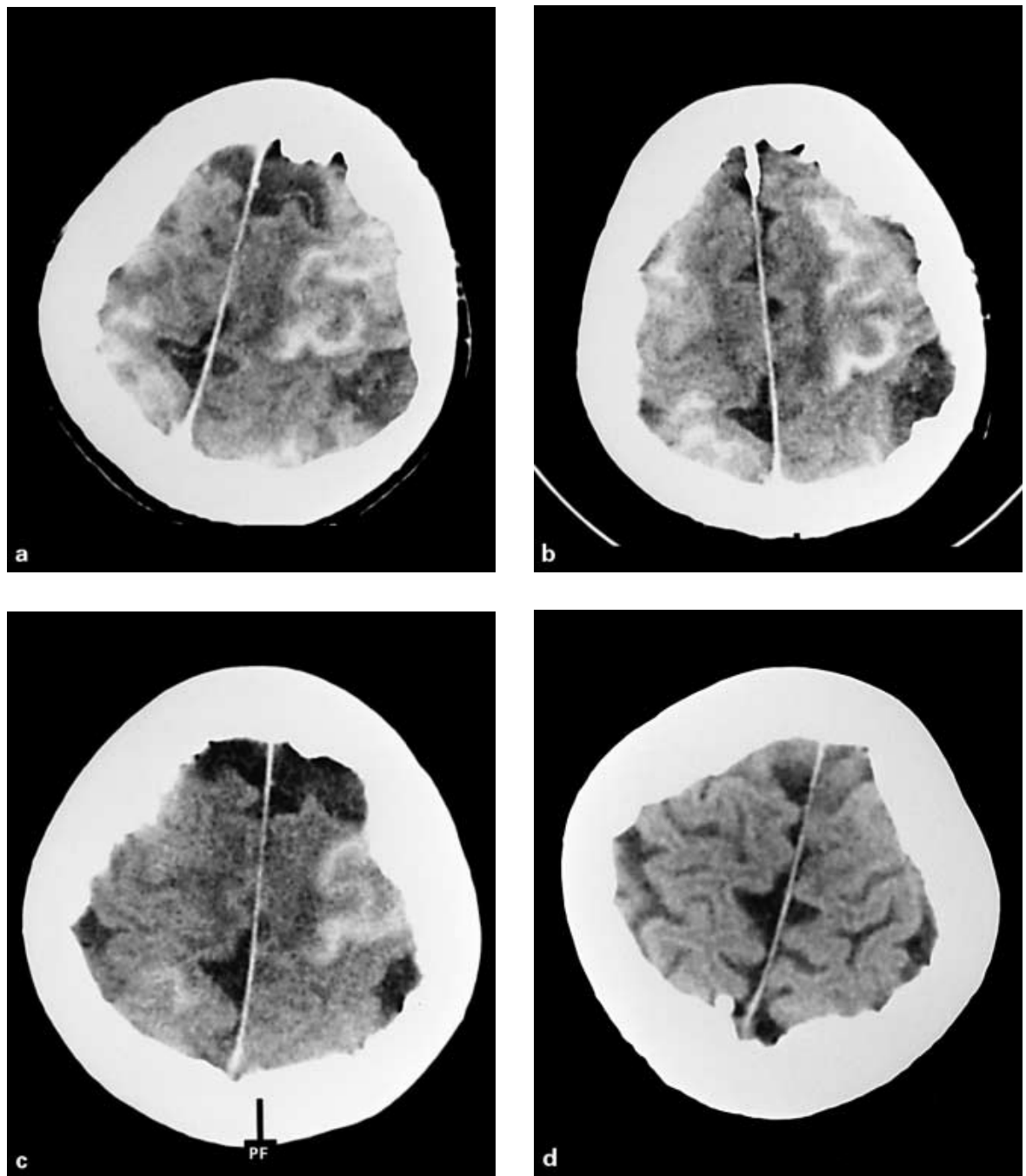

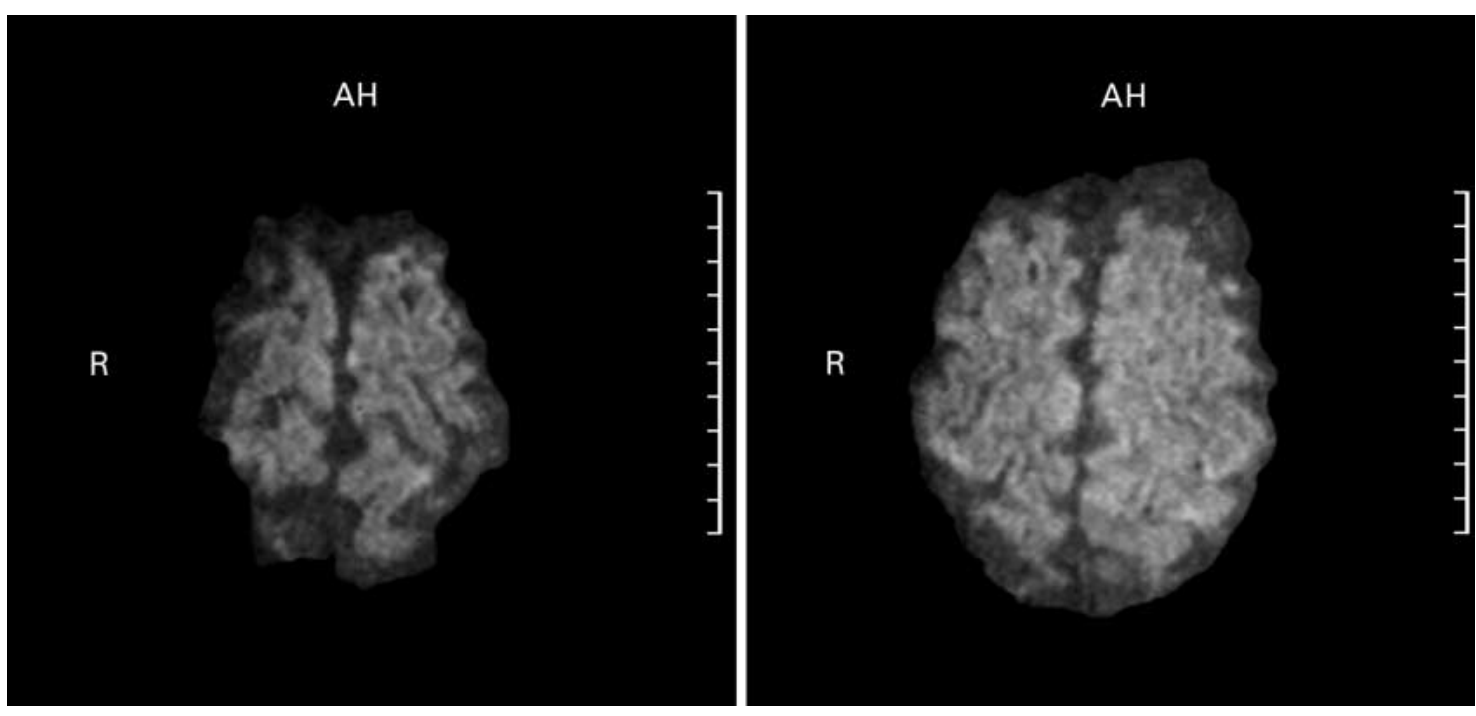

Fig. 2. Diffusion-weighted MRI with a b-value of 1,000 of the same anatomical region which displayed a contrast enhancement in the CCT. No sign of an acute or subacute stroke is visible.

procedure she became increasingly disoriented and drowsy. Her blood pressure rose to 200/100 $\mathrm{mm} \mathrm{Hg}$. Within the following $4 \mathrm{~h}$, she became aphasic and developed right hemiparesis. Cranial CT, performed first without and then with additional administration of $80 \mathrm{ml}$ Iomeron 300, revealed hyperdense material filling the sulci of both brain hemispheres, which was diagnosed as SAH (fig. 1a).

At this time the patient was referred to our acute stroke unit. On admission, $9 \mathrm{~h}$ postintervention, she exhibited severe global aphasia, mild to moderate right hemiparesis, meningism, confusion and somnolence. Blood pressure was $150 / 65 \mathrm{~mm} \mathrm{Hg}$. CT was repeated, confirming bihemispheric hyperdense filling of the cerebral sulci, which was more prominent on the left hemisphere (fig. 1b). Cranial Doppler ultrasonography detected no pathologic flow signals. Twenty-four hours postevent, lumbar CSF analysis yielded normal standard results concerning cell count and protein content; particularly neither xanthochromia nor red blood cells were seen. Cerebral MRI compiling T1, T2, gradient echo, FLAIR, proton density, spin echo, diffusion-weighted sequences and MR angiography excluded hemorrhage, ischemia, or any other significant pathology besides mild hypertensive microangiopathy and dilative arteriopathy. There was no gadolinium enhancement. Figure 2 shows the normal diffusion-weighted MRI images of the same regions displaying the CCT contrast enhancement. There was no sign of cerebral ischemia.

Spontaneous recovery was observed within $40 \mathrm{~h}$ postangiography. The patient fully regained consciousness, orientation, language ability, and muscular strength. In parallel, as observed with repeat CT scans, sulcal hyperdensities partly faded after $21 \mathrm{~h}$ (fig. 1c) and totally disappeared within $45 \mathrm{~h}$ (fig. 1d). Serum creatinine, which had risen to $141 \mu M$ intermediately, returned to baseline levels $(115 \mu M)$. The patient was monitored 3 more days before release. Follow-up interviews 1 and 4 weeks later confirmed that the patient had no sequelae other than persistent amnesia obscuring the 24-hour period immediately following angiography. Seizures were never observed.
Chemical assessment of iomeprol concentrations (kindly performed by Dr Lorusso, Bracco SpA) revealed a strong enrichment of iomeprol in the lumbar CSF $(547 \mu \mathrm{g} / \mathrm{ml})$ as compared to the simultaneous serum sample $(129 \mu \mathrm{g} / \mathrm{ml}) 24 \mathrm{~h}$ after coronary angiography. Voxel density measurements within the initial CT scan showed that the subarachnoidal hyperdensities reached up to 96 Hounsfield units.

\section{Discussion}

To our knowledge, this is the first case illustrating the occurrence of focal deficits such as hemiparesis and aphasia following coronary angiographic application of nonionic CM. Furthermore, our case shows that CT findings due to CM extravasation can be indistinguishable from $\mathrm{SAH}$, so that the correct diagnosis requires further investigations. As presented here, routine CSF analysis and MRI excluded SAH. Special CSF analysis confirmed iomeprol enrichment in the CSF as the cause of subarachnoid contrast enhancement. This was also consistent with voxel densities reaching as much as 96 Hounsfield units, clearly exceeding blood density ranging from 40 to 60 units [5]. CM enrichment in the CSF is a consequence of CMtoxic blood-brain/blood-CSF barrier disruption followed by $\mathrm{CM}$ extravasation [1]. A subacute stroke as the source of a disturbed blood-brain barrier could be excluded by the MRI findings with a normal diffusion-weighted image of the same anatomical region which displayed contrast enhancement in the sulci in the CCT

Although the administration of $500 \mathrm{ml}$ Iomeron 350 was just below the maximum recommended dose for coronary angiography as indicated by the manufacturer, it may be regarded as a relative overdosage in our patient with underlying chronic renal function impairment. Taking this into account, an additional dose, as given here for contrast CT, should have been avoided.

In conclusion, apparent SAH following any intravascular application of any CM has to be clarified by lumbar puncture and/or MRI, even if $\mathrm{CT}$ strongly suggests $\mathrm{SAH}$, in order to avoid additional $\mathrm{CM}$ 
administration and toxicity as well as further risks associated with cerebral angiography, which is required in the case of true SAH.

\section{References}

1 Torvik A, Walday P: Neurotoxicity of water-soluble contrast media. Acta Radiol Suppl 1995;399:221-229.

2 Lantos G: Cortical blindness due to osmotic disruption of the blood-brain barrier by angiographic contrast material: CT and MRI studies. Neurology 1989;39:567-571.

3 Sticherling C, Berkefeld J, Auch-Schwelk W, Lanfermann H: Transient bilateral cortical blindness after coronary angiography. Lancet 1998;351: 570.

4 Parry R, Rees JR, Wilde P: Transient cortical blindness after coronary angiography. Br Heart J 1993;70:563-564.

5 Sharp S, Stone J, Beach R: Contrast agent neurotoxicity presenting as subarachnoid hemorrhage. Neurology 1999;52:1503-1505.

6 May EF, Ling GSF, Geyer CA, Jabbari B: Contrast agent overdose causing brain retention of contrast, seizures, and parkinsonism. Neurology 1993; 43:836-838.

Prof. Gerhard Hamann, Department of Neurology Klinikum Grosshadern, Ludwig Maximilian University Marchioninistrasse 15, D-81377 Munich (Germany)

Tel. +49 8970953670 , Fax +49 8970953677

E-Mailhamann@brain.nefo.med.uni-muenchen.de 